\title{
$\begin{array}{ll}\text { Research Square } & \text { Preprints are preliminary reports that have not undergone peer review. } \\ \text { They should not be considered conclusive, used to inform clinical practice, }\end{array}$ or referenced by the media as validated information.
}

\section{Familial Hypocalciuric Hypercalcemia: The Challenge of Diagnosis}

\author{
David Taïeb ( $\square$ david.taieb@ap-hm.fr) \\ la timone university hospital \\ Adele Lasbleiz \\ AP-HM: Assistance Publique Hopitaux de Marseille \\ Nunzia Cinzia Paladino \\ AP-HM: Assistance Publique Hopitaux de Marseille \\ Pauline Romanet \\ AP-HM: Assistance Publique Hopitaux de Marseille \\ Frédéric Castinetti \\ AP-HM: Assistance Publique Hopitaux de Marseille \\ Thomas Cuny \\ AP-HM: Assistance Publique Hopitaux de Marseille \\ Frédéric Sebag \\ AP-HM: Assistance Publique Hopitaux de Marseille
}

\section{Research Article}

Keywords: Familial hypocalciuric hypercalcemia, parathyroid imaging, genetics, CaSR

Posted Date: August 6th, 2021

DOI: https://doi.org/10.21203/rs.3.rs-773127/v1

License: (c) (1) This work is licensed under a Creative Commons Attribution 4.0 International License. Read Full License 


\section{Abstract}

Familial hypocalciuric hypercalcemia $(\mathrm{FHH})$ is an autosomal dominant genetic disorder classically characterized by lifelong mild-to-moderate asymptomatic hypercalcemia with inappropriately normal to elevated serum parathyroid hormone (PTH) concentrations and hypocalciuria, best expressed by a urine calcium-to-creatinine clearance ratio (CCCR)<0.01[1,2]. FHH prevalence is estimated between 1:10 000 to 1:100 000[3,4]. In 60\% of cases, $\mathrm{FHH}$ is due to CASR inactivating mutation[5]. More rarely FHH is due to AP2S1 or GNA11 inactivating mutation, both genes encoding for proteins involved downstream of CASR activation[6]. These molecular alterations are found in all parathyroid cells, explaining disease persistence following partial parathyroidectomy and the ineffective surgical management of these patients. FHH phenotypes could however overlap with primary hyperparathyroidism (PHPT). Indeed, even if patients with FHH are currently asymptomatic, some of them present chondrocalcinosis, kidney stones or bone fracture and very high level of PTH or calcemia[7]. Nonetheless, the distinction has to be adressed since the therapeutic approach significantly differs between these two conditions. Surgery is usually recommended for PHPT[8] while follow-up is preferred in the latter case[9,10]. We report and discuss 7 cases, 6 out 7 being operated for a presumed PHPT.

\section{Introduction}

Familial hypocalciuric hypercalcemia $(\mathrm{FHH})$ is an autosomal dominant genetic disorder classically characterized by lifelong mild-to-moderate asymptomatic hypercalcemia with inappropriately normal to elevated serum parathyroid hormone (PTH) concentrations and hypocalciuria, best expressed by a urine calcium-to-creatinine clearance ratio (CCCR) < 0.01[1, 2]. FHH prevalence is estimated between 1:10 000 to 1:100 000[3, 4]. In 60\% of cases, FHH is due to CASR inactivating mutation[5]. More rarely FHH is due to AP2S1 or GNA11 inactivating mutation, both genes encoding for proteins involved downstream of CASR activation[6]. These molecular alterations are found in all parathyroid cells, explaining disease persistence following partial parathyroidectomy and the ineffective surgical management of these patients. FHH phenotypes could however overlap with primary hyperparathyroidism (PHPT). Indeed, even if patients with FHH are currently asymptomatic, some of them present chondrocalcinosis, kidney stones or bone fracture and very high level of PTH or calcemia[7]. Nonetheless, the distinction has to be adressed since the therapeutic approach significantly differs between these two conditions. Surgery is usually recommended for PHPT[8] while follow-up is preferred in the latter case[9, 10]. We report and discuss 7 cases, 6 out 7 being operated for a presumed PHPT.

\section{Methods}

We performed a retrospective analysis of patients with hyperparathyroidism managed in the department of Endocrine Surgery that were found to carry CASR variants between 2016-2020. Our surgery department is a high volume endocrine surgery deparment with approximately 300 parathyroid interventions per year. The study was approved by the Assistance Publique-Hôpitaux de Marseille ethics advisory committee (PADS21-46).

\section{Results}

Seven index cases (5F/2M, mean age 53.4y) with CaSR variants were identified, 6 of which had undergone surgical intervention. Six out of 7 could be classified as $\mathrm{FHH}$ with identification of new pathogenic or likely pathogenic variants, while the 7th case had a parathyroid adenoma (PA) with a CASR variant that was ultimately classified as variant of uncertain significance (VUS). All had hypercalcemia with inappropriately normal or high PTH 1-84 values (Table 1).

Three patients presented with a classical FHH phenotype. In the first case (1:F-45y), the disease occurred in a context of IgA nephropathy with progressive decline of kidney function. Surgical intervention was indicated after a 5 years follow-up in our nephrology tertiary referral center, where hypocalciuria was misinterpreted as being secondary to chronic kidney disease, in the presence of a single parathyroid abnormality visible on both neck ultrasound (US) and parathyroid scintigraphy. Calcemia was $2.64 \mathrm{mmol} / \mathrm{L}(\mathrm{N}: 2.20-2.55 \mathrm{mmol} / \mathrm{L})$ with high PTH level $(68.8 \mathrm{pg} / \mathrm{ml}, \mathrm{N}: 15-65 \mathrm{pg} / \mathrm{ml})$. Minimally invasive surgery was converted to open surgical intervention due to insufficient decrease of intraoperative PTH, and a subtotal parathyroidectomy (PTx) (3 glands removed) was performed.

The second patient (2:M-76y) had a previous history of resection of a parathyroid adenoma. His latest follow-up showed a persistent hypercalcemia $(2.65 \mathrm{mmol} / \mathrm{L})$ with hypophosphatemia $(0.75 \mathrm{mmol} / \mathrm{L}, \mathrm{N}: 0.81-1.45 \mathrm{mmol} / \mathrm{L})$. Calciuria was in the normal range limit (103 and 160mg/day,N:100$300 \mathrm{mg} /$ day) and his CCCR (0.017) was inconclusive to differentiate between FHH and PHPT. The patient had renal lithiasis, osteopenia and chronic renal failure stade 2. Neck US and parathyroid scintigraphy were negative. Because of the presumed persistent PHPT with negative parathyroid imaging, a genetic screening was performed. Genetic testing for case 2 revealed the same pathogenic CASR variant as case 1(c.893C>T,p.(Ala298Val))[7], a finding which argued against reoperation.

The third patient (3:M-77y) had a classical FHH biochemical phenotype (calcemia=2.61 mmol/L,PTH=70pg/mL,calciuria=72mg/day,CCCR=0.007). Imaging were negative. Genetic testing revealed a CASR pathogenic variant in exon 5(c.1525G>A,p.(Gly509Arg))[11].

Three additional cases had undergone surgical intervention due to a less typical clinical picture with marked hypercalcemia and fluctuating values of calciuria. All of them were relatively young at diagnosis and had negative parathyroid imaging studies despite significant hypercalcemia $(2.80,2.90,2.84 \mathrm{mmol} / \mathrm{L}$,respectively). Bilateral neck exploration was performed which resulted in resection of two hyperplastic glands in cases 4 and 6 , and one hyperplastic gland in case 5 . All had persistent hypercalcemia post-operatively. It has to be underlined that the existence of a multiglandular disease, 
objectivated during cervicotomy, prompted the surgeons to limit the extent of parathyroid resection to prevent the occurrence of post-operative

hypoparathyroidism. Genetic counseling enabled the identification of relatives with CASR variants.

In case 4 (F-42y), post-operative calciuria $(400 \mathrm{mg} / 24 \mathrm{~h})$ and CCCR $(0.021)$ were not decreased. Genetic testing detected a pathogenic CASR variant(c.1664T>C,p.(Ile555Thr))[7,12]. Her daughter, 20y, had a mild hypercalcemia (2.63mmol/L), hypophosphatemia (0.65mmol/L), mild elevated PTH $(73 \mathrm{pg} / \mathrm{mL})$, normal calciuria $(144 \mathrm{mg} / 24 \mathrm{~h})$ with a decreased CCCR $(0.008)$. Her son, 18y, had significant hypercalcemia (2.88mmol/L), normal PTH $(58 \mathrm{pg} / \mathrm{mL})$, normal calciuria $(171 \mathrm{mg} / 24 \mathrm{~h})$ and a decreased CCCR (0.006). Again, both children had negative parathyroid scintigraphy.

In another patient (5:F-36y) a CASR variant(c.511A>T,p.(Ser171Cys)) was identified. It was present in her mother, her sister, her brother and her niece. followed-up in our institution and has a marked hypercalcemia $(2.94 \mathrm{mmol} / \mathrm{L})$.

The 6th patient (6:F,52y) had a CASR variant(c.1664T>C,p.(Ile555Thr)). Her brother carried the same variant and had hypercalcemia (2.80mmol/L) with hypocalciuria $(68 \mathrm{mg} /$ day).

Taken together, these cases (4-6) illustrate that the presence of hypercalcemia in relatives during family screening is very powerful indicator suggesting for the diagnosis of $\mathrm{FHH}$. Negative parathyroid imaging together with marked hypercalcemia and calciuria in the low normal range could also raise the suspicion of $\mathrm{FHH}$.

Finally, the last case (7: F-46y) had typical features of PHPT: calcemia>3mmol/L with a marked elevation of serum PTH 1-84 (629pg/ml), hypophosphatemia $(0.74 \mathrm{mmol} / \mathrm{L})$ and a normocalciuria $(234 \mathrm{mg} /$ day) with a high CCCR $(0.026)$. Work-up revealed an osteopenia, a stage 3 renal insufficiency with normal kidney ultrasound. Neck US and parathyroid scintigraphy were concordant for a left P4-derived adenoma. A large left P4 adenoma, i.e adenoma on left superior parathyroid gland (weight:580mg), was resected via minimally-invasive surgery with a significant decline in intraoperative PTH 1-84. Genetic testing revealed an unknown CaSR variant in exon 3(c.347C>T,p.(Ala116Val)) classified as VUS. Phosphocalcic imbalance was normalized following surgery. Although, we could consider in the present case that CASR may be not involved in the patient's pathology, physicians should be aware that this rare variant may be not always pathogenic.

\section{Discussion}

This series illustrates the heterogeneity in FHH biochemical phenotypes with a possible overlap with PHPT that could lead to inappropriate management. Beyond biochemical characterization, parathyroid imaging also plays an important role in parathyroid disease subtyping. In PHPT, a negative parathyroid imaging study should raise the suspicion of multiglangular hyperplasia[13,14]. This series shows that negative parathyroid imaging is part of the clinical picture of $\mathrm{FHH}$. In France, FHH genetic testing is recommended in all patients presenting with familial PHPT or isolated PHPT before 50 years old. This involves the simultaneous exploration of genes involved syndromic hyperparathyroidism (i.e.MEN1, CDKN1B, CDC73)[15]. Genetic testing should be also performed in case of suspicion of FHH or in doubtful clinical situations or in patients with persistent hypercalcemia following PTx. However, despite technological improvements in genetic sequencing, it does not appear reasonable to submit all hyperparathyroid patients to genetic analysis. In our insitution, we are in agreement with the guidelines that recommend performing genetic testing for patients with one of the following criteria[16]:

- Age<50y

- Family history of hypercalcemia

- Hyperparathyroidism with CCCR<0.01 and/or calciuria below $1.33 \mathrm{mg} / \mathrm{day} / \mathrm{kg}$ using 24-hour urine collection

However, based on this case series, we would like to emphasize that age at diagnosis has a limited diagnostic value, presence of single gland abnormality on parathyroid imaging is not synonym of PHPT and presence of CASR variant is not synonym of FHH.

Addtionally, we consider that :

1. FHH screening is unnecessary if simultaneous calciuria and CCCR are above $4 \mathrm{mg} / \mathrm{kg} / 24 \mathrm{~h}$ and 0.02 , respectively. Indeed, looking specifically at CCCR and $24 \mathrm{~h}$-calciuria, we found very few patients $[7,17]$ with a real FHH (i.e.without single parathyroid adenoma) and simultaneous CCCR>0.02 and $24 \mathrm{~h}$-calciuria $>0.1 \mathrm{mmol} / \mathrm{Kg}$

2. In the presence of serum total calcium $\geq 2.8 \mathrm{mmol} / \mathrm{L}$, a negative parathyroid imaging should raise the suspicion of $\mathrm{FHH}$.

3. In case of doubt regarding the disease, assessement of calcemia in first degree relatives is of powerful value and may guide genetic testing.

4. HPT patients, especially this negative or discordant imaging findings, should be discussed at a multidisciplinary team meeting in order to ensure proper management.

\section{Declarations}

\section{Funding}

This research was not funded by a specific project grant

\section{Declaration of interest}


The authors have declared no conflicts of interest.

\section{References}

1. Arshad MF, McAllister J, Merchant A et al. Urinary calcium indices in primary hyperparathyroidism (PHPT) and familial hypocalciuric hypercalcaemia (FHH): which test performs best? Postgrad. Med. J. (2020).

2. Lee JY, Shoback DM. Familial hypocalciuric hypercalcemia and related disorders. Best Pract. Res. Clin. Endocrinol. Metab. 32(5), 609-619 (2018).

3. Christensen SE, Nissen PH, Vestergaard P, Mosekilde L. Familial hypocalciuric hypercalcaemia: a review. Curr. Opin. Endocrinol. Diabetes Obes. 18(6), 359-370 (2011).

4. Dershem R, Gorvin CM, Metpally RPR et al. Familial Hypocalciuric Hypercalcemia Type 1 and Autosomal-Dominant Hypocalcemia Type 1: Prevalence in a Large Healthcare Population. Am. J. Hum. Genet. 106(6), 734-747 (2020).

5. Vargas-Poussou R, Mansour-Hendili L, Baron S et al. Familial Hypocalciuric Hypercalcemia Types 1 and 3 and Primary Hyperparathyroidism: Similarities and Differences. J. Clin. Endocrinol. Metab. 101(5), 2185-2195 (2016).

6. Nesbit MA, Hannan FM, Howles SA et al. Mutations affecting G-protein subunit a11 in hypercalcemia and hypocalcemia. N. Engl. J. Med. 368(26), 2476-2486 (2013).

7. Mouly C, Vargas-Poussou R, Lienhardt A et al. Clinical characteristics of familial hypocalciuric hypercalcaemia type 1: A multicentre study of 77 adult patients. Clin. Endocrinol. (Oxf.) 93(3), 248-260 (2020).

8. National Guideline Centre (UK). Hyperparathyroidism (primary): diagnosis, assessment and initial management. National Institute for Health and Care Excellence (UK), London (2019).

9. Cristina E-V, Alberto F. Management of familial hyperparathyroidism syndromes: MEN1, MEN2, MEN4, HPT-Jaw tumour, Familial isolated hyperparathyroidism, FHH, and neonatal severe hyperparathyroidism. Best Pract. Res. Clin. Endocrinol. Metab. 32(6), 861-875 (2018).

10. Vahe C, Benomar K, Espiard S et al. Diseases associated with calcium-sensing receptor. Orphanet J. Rare Dis. 12 (2017).

11. Nissen PH, Christensen SE, Heickendorff L, Brixen K, Mosekilde L. Molecular genetic analysis of the calcium sensing receptor gene in patients clinically suspected to have familial hypocalciuric hypercalcemia: phenotypic variation and mutation spectrum in a Danish population. J. Clin. Endocrinol. Metab. 92(11), 4373-4379 (2007).

12. Tonyushkina KN, O'Connor S, Dunbar NS. A novel CaSR mutation presenting as a severe case of neonatal familial hypocalciuric hypercalcemia. Int. J. Pediatr. Endocrinol. 2012(1), 13 (2012).

13. Sebag F, Hubbard JGH, Maweja S, Misso C, Tardivet L, Henry J-F. Negative preoperative localization studies are highly predictive of multiglandular disease in sporadic primary hyperparathyroidism. Surgery 134(6), 1038-1041; discussion 1041-1042 (2003).

14. Hindié E, Zanotti-Fregonara P, Tabarin A et al. The role of radionuclide imaging in the surgical management of primary hyperparathyroidism. J. Nucl. Med. Off. Publ. Soc. Nucl. Med. 56(5), 737-744 (2015).

15. Thakker RV. Genetics of parathyroid tumours. J. Intern. Med. 280(6), 574-583 (2016).

16. Eastell R, Brandi ML, Costa AG, D'Amour P, Shoback DM, Thakker RV. Diagnosis of asymptomatic primary hyperparathyroidism: proceedings of the Fourth International Workshop. J. Clin. Endocrinol. Metab. 99(10), 3570-3579 (2014).

17. Gunn IR, Wallace JR. Urine calcium and serum ionized calcium, total calcium and parathyroid hormone concentrations in the diagnosis of primary hyperparathyroidism and familial benign hypercalcaemia. Ann. Clin. Biochem. 29 ( Pt 1), 52-58 (1992).

\section{Tables}

Table 1 - Clinical, biological, imaging and genetic characteristics of FHH patients 
Case 2

Case 3

Case 6

Case 7

\begin{tabular}{ll} 
Sex & F \\
\hline Age (years) & 45 \\
\hline $\begin{array}{l}\text { Previous familial history of } \\
\text { hypercalcemia }\end{array}$ & No \\
\hline Relative cases & No \\
\hline Comorbidities & $\begin{array}{l}\text { IgA } \\
\text { nephropathy }\end{array}$ \\
\hline Medications & ACE inhibitor \\
\hline
\end{tabular}

Weight (kg)

Calcium pre-operative (mmol/L)

Calcium post-operative

M

$\mathrm{M} \quad \mathrm{F}$

76

No

$77 \quad 42$

No

No

T2D

Metformin, IDPP4

50

Phosphore pre-

operative(mmol/L)

Phosphore post-operative

2.50

0.88

75

2.69

2.65

NA

0.75

39

vitamin D (nmol/L)

1.34

119

PTH pre-operative $(\mathrm{pg} / \mathrm{mL})$

68.8

PTH post operative $(\mathrm{pg} / \mathrm{mL}) \quad 98$

PTH per-operative $(\mathrm{pg} / \mathrm{mL})$

$98 \quad 97$

Creatinine (mcmol/L)

eGFR (mL/min)

Calciuria (mg/day)

Calciuria (mg/kg/day)

CCCR

Osteopenia/osteoporosis

Femoral T-score

L1-L4 T-score

Renal lithiasis

Neck ultrasound

$70.7->24.5$

NA

116

$49 \quad 66$

$102 \quad 73$

66

103

1.37

0.38

0.017

0.004

0.017

No Yes

0.4

1.2

No

Left P3

Parathyroid scintigraphy

Histopathology Uniglandular/Multiglandular
disease

CASR Mutation - Exon

Nucleotide variant

Protein variant

$\begin{array}{ll}\text { Protein variant } \quad \text { p.(Ala298Val) } & \text { p. } \\ & \end{array}$

$-1.1 \quad 0.4$

0

$-1.4$

Yes

Left P3

Hyperplasia

Multiglandular

CaSR exon $4 \quad$ CaSR exon 4

$\begin{array}{ll}\text { c.893C >T } & \text { c.893C>T } \\ \text { p.(Ala298Val) } & \begin{array}{l}\text { p. } \\ \text { (Ala298Val) }\end{array}\end{array}$

heterozygous heterozygous

Probably

pathogenic pathogenic Pathogenic
Probably pathogenic pathogenic Pathogenic

No No

No No

No Yes Yes Yes No

T2D, HTA No

Sulfuronylure- No No

Calcic

inhibitor

ARA2

$82 \quad 54$

2.61

$54 \quad 54$

54

2.90

2.75

2.83

0.57

0.77

45

45

98

93,1

216->67.9

56.1

112

183

3.32

0.80

80

42.4

71

96.2 -> 43.4

57

116

106

1.96

NA NA

NA

0.1

$-1.5$

NA

No

No

No Yes

$\begin{array}{lll}\text { NA } & \text { NA } & -1.1 \\ \text { NA } & \text { NA } & -0.4\end{array}$

Status

Classification
Negative

Yes

Negative

Negative

Hyperplasia

Hyperplasia

Multiglandular

Uniglandular

CaSR exon $5 \quad$ CaSR exon $6 \quad$ Cas

c. $1525 \mathrm{G}>\mathrm{A}$

c. $1664 \mathrm{~T}>\mathrm{C}$

CaSR exon 4

c. $511 \mathrm{~A}>\mathrm{T}$

p.

p.(lle555Thr)

p.(Ile555Thr)

(Ala116Val)

heterozygous heterozygous

$\begin{array}{ll}\text { Pathogenic } & \begin{array}{l}\text { Probably } \\ \text { Pathogenic }\end{array}\end{array}$

heterozygous heterozygous

Pathogenic Unknown 
T2D : Diabete type 2 , HTA : Hypertension

eGFR : estimated glomerular filtration rate, calculated according to the CKD-EPI formula.

CCCR : Calcium creatinine clearance ratio.

Previous family history of hypercalcemia was assessed by interwiewing index case and relative-cases were determined by performing phosphocalcic blood test and genetic research after detection of the mutation on the index case.

Femoral T-score is referring to femoral neck T-score.

P3 is defined by inferior parathyroid gland, P4 is defined by superior parathyroid gland

Normal range of biological value : Calcemia (2.20-2.55 mmol/L) - Phosphore (0.81-1.45mmol/L) - PTH (15-65 pg/ml) - Vitamin D (75-250 nmol/L) Calciuria (100-300mg/day)

\section{Supplementary Files}

This is a list of supplementary files associated with this preprint. Click to download.

- CaSRsuppldatarevised.docx 